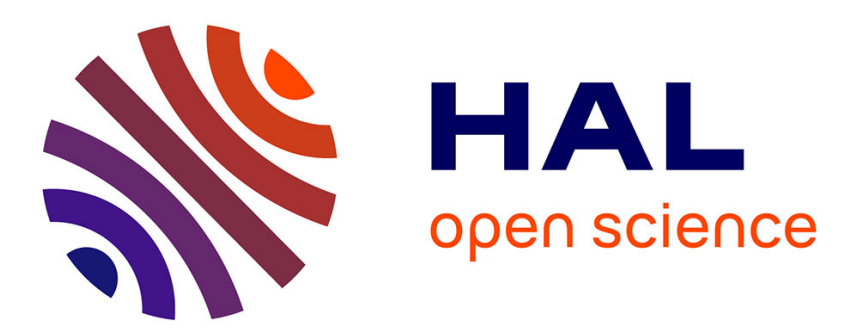

\title{
Appareillage d'écho de spin pour l'étude de la résonance magnétique nucléaire dans les substances ferro- et ferrimagnétiques
}

Le Dang Khoi

\section{- To cite this version:}

Le Dang Khoi. Appareillage d'écho de spin pour l'étude de la résonance magnétique nucléaire dans les substances ferro- et ferrimagnétiques. Revue de Physique Appliquée, 1968, 3 (2), pp.193-198. 10.1051/rphysap:0196800302019300 . jpa-00242844

HAL Id: jpa-00242844

https://hal.science/jpa-00242844

Submitted on 1 Jan 1968

HAL is a multi-disciplinary open access archive for the deposit and dissemination of scientific research documents, whether they are published or not. The documents may come from teaching and research institutions in France or abroad, or from public or private research centers.
L'archive ouverte pluridisciplinaire HAL, est destinée au dépôt et à la diffusion de documents scientifiques de niveau recherche, publiés ou non, émanant des établissements d'enseignement et de recherche français ou étrangers, des laboratoires publics ou privés. 


\title{
APPAREILLAGE D'ÉGHO DE SPIN POUR L'ÉTUDE DE LA RÉSONANGE MAGNÉTIQUE NUGLÉAIRE DANS LES SUBSTANGES FERRO- ET FERRIMAGNÉTIQUES
}

\author{
Par LE DANG KHOI, \\ Institut d'Électronique Fondamentale, Faculté des Sciences, Bâtiment 220, 91-Orsay. \\ (Reçu le 18 janvier 1968, révisé le 12 févriev 1968.)
}

\begin{abstract}
Résumé. - On décrit un appareillage d'écho de spin spécialement conçu pour l'étude de la résonance nucléaire dans les corps ferro- et ferrimagnétiques. Les principes de mesure des temps de relaxation $T_{1}$ et $T_{2}$, de la largeur de raie, du coefficient d'amplification $\eta$ sont donnés. On calcule le rapport signal sur bruit en tenant compte du "bruit ferromagnétique " dû aux fluctuations thermiques de l'aimantation spontanée. Cet appareillage qui fonctionne dans la gamme 20-150 MHz est caractérisé par sa sensibilité et sa maniabilité.
\end{abstract}

Abstract. - A spin-echo apparatus of N.M.R. in ferro- and ferrimagnetic materials is described. The methods for measuring the relaxation times $T_{1}$ and $T_{2}$, the line width, the enhancement factor $\eta$ are explained. The expression of the signal over noise ratio, including "ferromagnetic noise" due to the thermal fluctuations of spontaneous magnetization, is established. Throughout the usefull frequency range, $20-150 \mathrm{Mc} / \mathrm{s}$, this apparatus is characterized by its sensitivity and its high time resolution.

1. Introduction. - L'une des méthodes en résonance magnétique nucléaire est la méthode des échos de spin [1] qui, appliquée aux substances magnétiques, présente de nombreux avantages :

- Elle permet de détecter les raies très larges d'origine inhomogène, ce qui est fréquent dans les corps ferrimagnétiques.

- Sa sensibilité dépend peu de la fréquence tant que le bruit de la bobine est supérieur à celui du tube d'entrée.

- Elle permet de mesurer commodément non seulement les temps de relaxation, mais aussi la largeur de raie et le coefficient d'amplification $\eta$.

2. Principe de mesure. - Si l'on applique un champ de radiofréquence d'amplitude $2 H_{1}$ oscillant à la fréquence de Larmor, pendant un temps $t_{\mathrm{w}}$ court par rapport à $T_{2}$, l'aimantation nucléaire $m$ pivote autour de $H_{1}$ d'un angle $\theta=\gamma H_{1} t_{\mathrm{w}}$ où $\gamma$ est le rapport gyromagnétique nucléaire.. Une impulsion est dite de type $\pi / 2$ ou $\pi$ si $\theta=\pi / 2$ ou $\pi$.

$\mathrm{Si}$ au bout du temps $\tau$, après une impulsion de $\pi / 2$, on applique une impulsion de $\pi$, il est facile de montrer que tous les spins seront ramenés en phase à l'ins-

(1) Cet article recouvre la partie technique du travail d'une thèse de Doctorat d'Etat ès Sciences Physiques devant être soutenue à la Faculté des Sciences d'Orsay, au début de l'année 1968, et enregistrée sous le nº A.O. 1951 au C.N.R.S. tant $2 \tau$ : le signal d'induction s'appelle « l'écho de spin » dont la forme peut être considérée comme la juxtaposition de deux courbes de précession libre.

2.1. Mesure DE $T_{2}$ ET DE $T_{2}^{*}$. La constante de temps $T_{2}^{*}$ de la précession libre est liée à la largeur de raie $\Delta \nu$ par $T_{2}^{*}=1 / 2 \pi \Delta \nu$. La mesure de $T_{2}^{*}$ par la précession libre est imprécise car $T_{2}^{*}$ est en général très court. Par contre, on déduit facilement $T_{2}^{*}$ de la largeur $\Delta t$ de l'écho (à mi-hauteur) et qui vaut :

$$
\Delta t=2 \log _{\mathrm{e}} 2 T_{2}^{*}
$$

Cette formule permet de mesurer avec précision la largeur des raies fines, c'est-à-dire au cas où $T_{2}^{*} \gg 1 \mu \mathrm{s}$. Dans le cas où $T_{2}^{*} \ll 1 \mu \mathrm{s}$, il faut mesurer l'amplitude de l'écho en fonction de la fréquence.

L'amplitude de l'écho décroît exponentiellement en fonction du temps $\tau$ avec une constante de temps $T_{2}$, car les spins perdent leur mémoire de phase par relaxation transversale.

2.2. MEsure DE $T_{1}$. - Si on applique une impulsion de $\pi$ suivie d'une impulsion de $\pi / 2$ après un temps $\tau$, la précession libre $V(\tau)$ après celle-ci [2] varie suivant la relation :

$$
V(\tau)=V_{\max }\left[1-2 \exp \left(-\tau / T_{1}\right)\right] .
$$

Mais il est difficile de mesurer l'amplitude initiale du signal de précession libre qui est mélangé à la deuxième impulsion. Les conditions deviennent plus 
favorables si nous appliquons une troisième impulsion de $\pi$ juste après celle de $\pi / 2$ : ainsi on isole dans l'amplitude bien définie de l'écho la grandeur $V(\tau)$.

2.3. Mesure du faGteur D'Amplification. - Dans les expériences de résonance magnétique nucléaire, le noyau voit un champ de radiofréquence apparent $H_{\mathrm{x}}^{*}$ $\eta$ fois plus grand [3] que le champ appliqué $H_{\mathbf{x}}$. On définit donc le facteur d'amplification $\eta$ par :

$$
\eta=H_{x}^{*} / H_{x} \text {. }
$$

Pour une impulsion de $\pi / 2$, le produit $H_{x}^{*} t_{\mathrm{w}}$ sera tel que :

d'où :

$$
\pi / 2=\gamma H_{x}^{*} t_{\mathrm{w}} / 2
$$

$$
\eta=\frac{1}{2 \nu t_{\mathrm{w}}} \cdot \frac{H_{n}}{H_{x}}
$$

où $\nu$ est la fréquence de résonance nucléaire dans le champ $H_{n}$.

On mesure la largeur de l'impulsion de radiofréquence à l'oscilloscope et on déduit $H_{x}$ à partir des caractéristiques de la bobine. Soit $H_{x} \mathrm{e}^{j \omega_{n} t}$ le champ de radiofréquence à la fréquence angulaire $\omega_{n}=2 \pi \nu$ supposé uniforme dans une bobine de section $S$ ayant $n$ tours, le flux à travers la bobine est donné par :

$$
\varphi=n \mu S H_{x} \mathrm{e}^{j \omega_{n} t}
$$

où $\mu$ est la perméabilité apparente de l'échantillon.

La tension $V$ recueillie aux bornes de la bobine est donc :

ou :

$$
V=\mathrm{d} \varphi / \mathrm{d} t
$$

$$
\begin{gathered}
V_{0}=|V|=n \mu S H_{x} \omega_{n} \\
H_{x}=V_{0} / \mu n S \omega_{n} .
\end{gathered}
$$

La perméabilité apparente $\mu$ est égale à $\left(\omega_{0} / \omega_{n}\right)^{2}$ où $\omega_{0}$ est la fréquence angulaire du circuit oscillant à vide.

3. Intensité du signal nucléaire. - 3.1. TENSION INDUITE PAR LE SIGNAL NUGLÉAIRE. - Le signal déţecté est dû à la variation du moment magnétique électronique $M_{x}$ induit par le signal nucléaire $m_{x}$ par suite du couplage hyperfin. On peut montrer que le rapport $M_{x} / m_{x}$ est justement égal au facteur $\eta$.

La f.é.m. induite par l'aimantation nucléaire tournant $m=m_{0} \mathrm{e}^{i \omega_{n} t}$ dans une bobine de $n$ tours et de section $S$ s'écrit, en tenant compte du facteur $\eta$ :

$$
e=\eta 4 \pi n S m_{0} \omega_{n}
$$

en adoptant le couplage relatif à un milieu infini.

L'aimantation nucléaire macroscopique de l'échantillon est la même [4] que celle déduite de la susceptibilité statique nucléaire :

$$
m_{0}=\frac{N \gamma^{2} \hbar^{2} I(I+1)}{3 k T} H_{n}
$$

où $N$ est le nombre de spins nucléaires $I$ par unité de volume.

Si $\xi$ est le remplissage défini par le rapport du volume effectif de l'échantillon sur le volume de la bobine, la tension recueillie aux bornes du circuit oscillant de surtension $Q$ sera :

$$
V_{\mathrm{s}}=\xi . Q . e \text {. }
$$

3.2. ORIGINE DU BRUIT DE FOND. - Nous supposons que le bruit du tube d'entrée est négligeable devant celui de la sonde H.F. constituée d'un circuit oscillant L.C. Ge bruit est lié aux pertes dues à la résistance H.F. de la bobine d'une part, et à la susceptibilité $\chi^{\prime \prime}$ de l'échantillon ferromagnétique d'autre part. Ces résistances de pertes s'expriment en fonction de la surtension à vide $Q_{0}$, de la self à vide $L_{0}$, de la pulsation d'accord $\omega$, du coefficient de remplissage $\xi$ et de la susceptibilité $\chi^{\prime \prime}$ :

$$
\begin{aligned}
& r_{1}=L_{0} \omega / Q_{0} \\
& r_{2}=4 \pi \xi \chi^{\prime \prime} L_{0} \omega
\end{aligned}
$$

pour la bobine et l'échantillon respectivement.

La surtension en charge $Q$ est déterminée par :

$$
\begin{aligned}
& Q=\mu L_{0} \omega /\left(r_{1}+r_{2}\right) \\
& Q=\mu Q_{0} /\left(1+Q_{0} 4 \pi \xi \chi^{\prime \prime}\right)
\end{aligned}
$$

où $\mu$ est la perméabilité apparente de l'échantillon.

On recueille donc aux bornes du circuit oscillant de bande passante $\Delta f$ une tension de bruit :

$$
V_{\mathrm{B}}=Q \sqrt{4 k T \Delta f\left(L_{0} \omega / Q_{0}\right)\left(1+Q_{0} 4 \pi \xi \chi^{\prime \prime}\right)}
$$

avec $\Delta f=\omega / 2 \pi Q$ :

$$
V_{\mathbf{B}}=2 \omega \sqrt{\mu L_{0} k T / 2 \pi} .
$$

On en déduit le rapport signal sur bruit :

$$
\frac{V_{\mathrm{S}}}{V_{\mathrm{B}}}=\frac{\xi \cdot Q \cdot \eta \cdot(2 \pi)^{3 / 2} n S \omega_{n} N \gamma \hbar^{2} I(I+1)}{3(k T)^{3 / 2}\left(\mu L_{0}\right)^{1 / 2}} .
$$

Naturellement, cette expression suppose que la bande passante $B$ du récepteur est au moins égale à $\Delta f$. Si cette condition n'est pas remplie, on doit remplacer $\Delta f$ par $B$.

Comme le facteur $\eta$ est proportionnel à $H_{n}$, on peut écrire le rapport signal sur bruit sous la forme :

$$
\frac{V_{\mathrm{S}}}{V_{\mathrm{B}}}=\frac{(2 \pi)^{3 / 2} a \xi \cdot Q \cdot n \cdot S \cdot N \cdot \hbar^{2} I(I+1) \omega_{n}^{2}}{3(k T)^{3 / 2}\left(\mu L_{0}\right)^{1 / 2}}
$$

où $a$ est un coefficient qui dépend des propriétés magnétiques de l'échantillon.

4. Appareillage expérimental. - 4.1. Problèmes PARTIGULIERS A LA RÉSONANGE NUGLÉAIRE DANS LES FERROMAGNÉTIQUES. - Si, dans un appareillage d'échos de spin classique, on peut se contenter d'impulsions de radiofréquence de longueur 10 à $20 \mu \mathrm{s}$, nous avons besoin ici d'impulsions beaucoup plus fines (de l'ordre d'une microseconde) à cause de la petitesse de $T_{2}^{*}$. Par contre, le champ de radiofréquence n'a pas besoin d'être très important car on bénéficie de l'effet d'amplification.

La principale difficulté dans la réalisation d'un appareillage approprié est que celui-ci doit être faci- 
lement réglable en fréquence, car la fréquence de résonance nucléaire est essentiellement une variable dans l'étude des substances ferromagnétiques. Cela veut dire que l'émetteur ne doit comporter qu'un circuit résonnant qui sera le circuit d'entrée du récepteur. Le problème ainsi posé n'est pas simple si l'on tient compte des difficultés secondaires, comme les pertes H.F. causées par l'échantillon ferromagnétique, etc.

4.2. RÉAlisation de L'Appareillage. - Nous avons réalisé un appareillage d'échos de spin en tenant compte de toutes ces données. Nous nous sommes efforcés de trouver des compromis optimaux partout où les exigences a priori se trouvaient en contradiction. Ge genre de problème est fréquent en électronique et la solution optimale ne peut être trouvée que dans l'étude expérimentale.

4.2.1. Schéma d'ensemble de l'appareillage. - La figure 1 représente le schéma d'ensemble de l'appareillage. Le générateur de dents de scie de fréquence

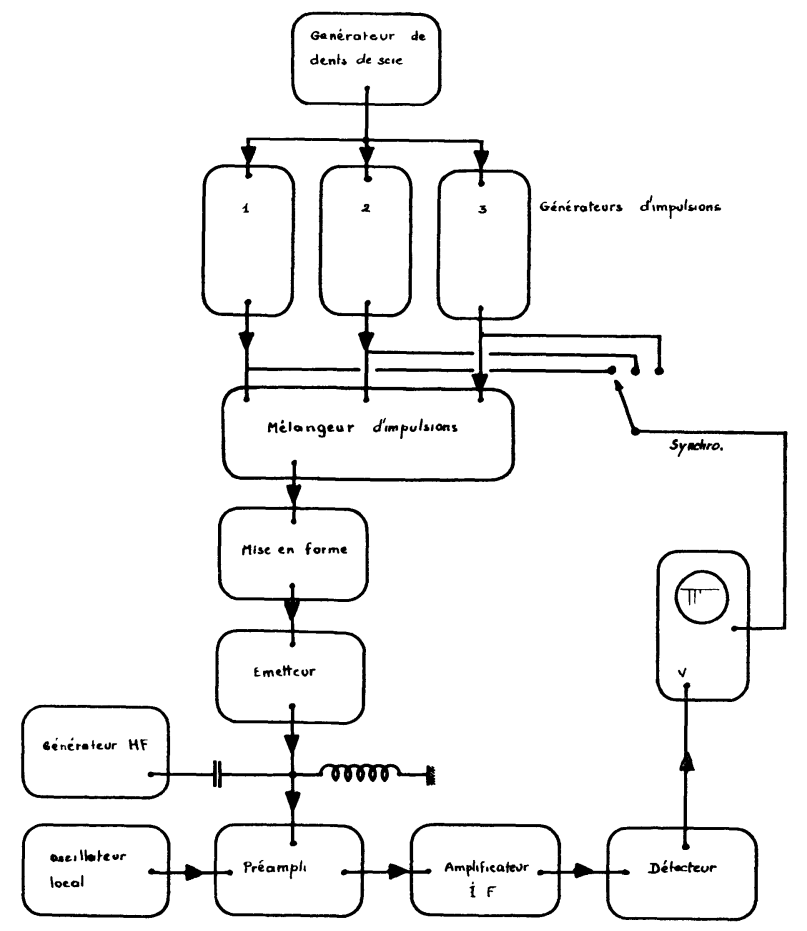

FIG. 1.

variable sert à régler la période des séquences d'impulsions de façon que celle-ci soit grande par rapport à $T_{1}$. Il synchronise les générateurs d'impulsions de largeur et de phase réglables. Ces impulsions sont mélangées, amplifiées et mises en forme avant d'être appliquées à un oscillateur pulsé. Le signal nucléaire est d'abord amplifié par un tube à faible bruit, à large bande passante. Cet étage est suivi d'un dispositif convertisseur de fréquence et d'un amplificateur de radiofréquence. Après détection, le signal est envoyé à un oscilloscope H.F. qui est synchronisé par l'une des trois impulsions. De cette façon, on peut observer en détail les impulsions de radiofréquence et l'écho.

4.2.2. Réalisation pratique. - Hormis l'oscillateur pulsé, les autres parties importantes du montage sont le circuit de mise en forme des impulsions et le préamplificateur avec le convertisseur de fréquence, le reste étant relativement classique. Pour améliorer la possibilité de mesurer des temps $T_{2}$ très courts, nous avons construit en outre un dispositif permettant de supprimer la traînée des impulsions de radiofréquence. Nous ne décrivons que les parties originales de cet appareillage.

a) Oscillateur. - Le problème est de trouver un oscillateur qui remplisse les conditions suivantes :

1. Posséder un seul circuit résonnant pour pouvoir facilement régler la fréquence.

2. Avoir un niveau d'oscillation relativement élevé en présence de l'échantillon.

3. Pouvoir produire des impulsions de radiofréquence très fines.

4. Fonctionner correctement jusqu'à une centaine de mégahertz.

5. Être facilement utilisable pour l'étude en fonction de la température et du champ magnétique extérieur.

Après avoir étudié plusieurs types d'oscillateurs tels que oscillateur à couplage grille-cathode, oscillateur à couplage capacitif grille-anode avec les différents

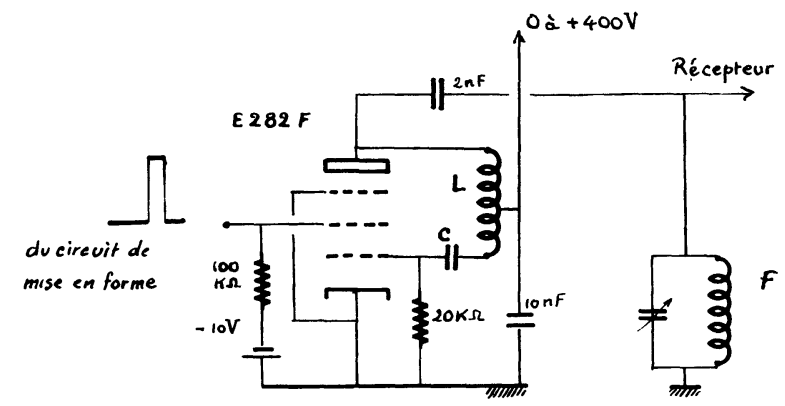

FIG. 2.

tubes électroniques, nous avons trouvé une solution satisfaisante dans le montage ci-contre (fig. 2).

C'est un oscillateur à couplage selfique grille-anode. La réaction positive est assurée par la self $L$ qui, ayant un point à la masse H.F., fonctionne comme un transformateur entre grille et anode. L'écran est polarisé négativement pour bloquer complètement le tube en l'absence d'impulsion. Il y a une valeur de $C$ pour laquelle l'oscillation est optimale : si $C$ est trop petit, la tension injectée sur la grille est trop faible à cause de la capacité d'entrée grille-cathode; si $C$ est trop grand, l'oscillation est amortie par la résistance d'entrée du tube. 


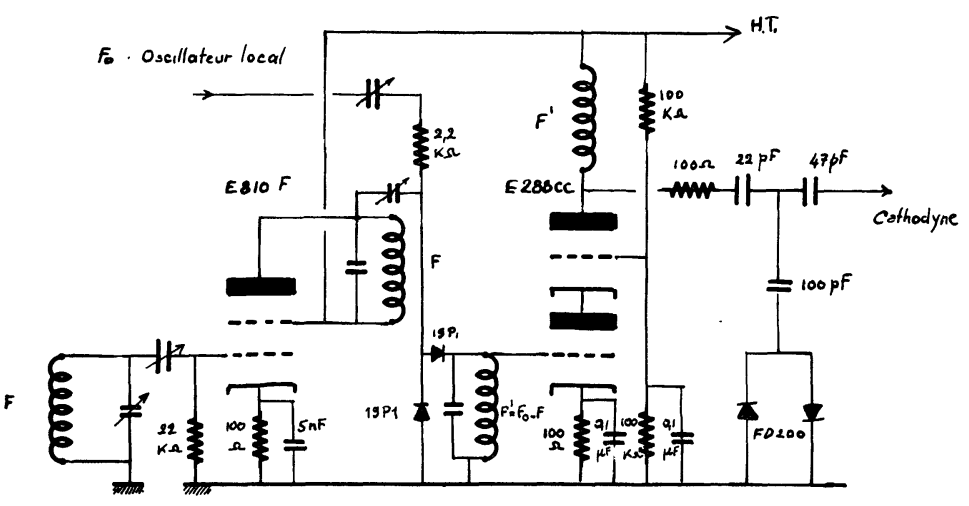

FIG. 3.

Cet oscillateur remplit toutes les cinq conditions énumérées ci-dessus moyennant quelques précautions :

- Le tube électronique doit être une pentode H.F. à grande pente et à faible conductance d'entrée.

- La self $L$ doit être grande devant la self du circuit résonnant mais avoir en même temps un facteur de surtension suffisant pour assurer la réaction. Pour couvrir toute la gamme de fréquence, nous disposons d'un jeu de bobines embrochables.

- Les connexions doivent être aussi courtes que possible. Toutefois, on peut relier sans inconvénient le circuit oscillant à la plaque du tube par un câble coaxial, ce qui rend le montage très pratique.

Remarquons que, lorsque le tube est au cut-off, l'amortissement du circuit oscillant est faible et par conséquent il est alors possible d'utiliser ce circuit comme circuit d'entrée du préamplificateur, pour détecter le signal nucléaire dans une expérience d'écho de spin.

b) Préamplificateur. - Nous avons construit un préamplificateur à très faible bruit et dont il est facile de changer la fréquence d'accord. Le schéma se comprend de lui-même ( fig. 3). Le circuit oscillant n'est pas relié directement à la grille du tube d'entrée, mais on intercale un très faible condensateur (environ $10 \mathrm{pF}$ ), afin de conserver un niveau d'oscillation élevé et un bon facteur de surtension. La bande passante du circuit plaque $F$ est choisie assez large (une dizaine de mégahertz) pour faciliter la recherche des signaux inconnus. Ceci nécessite bien entendu un tube ayant un grand facteur de mérite. L'opération d'hétérodynage est d'autant meilleure que la fréquence $F^{\prime}$ est plus petite, mais la détection de l'écho est mauvaise si $F^{\prime}$ n'est pas grand devant la largeur du spectre de l'écho. Nous avons choisi un compromis en prenant $F^{\prime}=15 \mathrm{MHz}$ avec une bande passante de $2 \mathrm{MHz}$. En outre, nous évitons la saturation de l'amplificateur H.F. par les impulsions de radiofréquence au moyen d'un limiteur à diodes, ce qui est facile à $15 \mathrm{MHz}$. La sortie cathodyne est classique. Ici encore nous disposons de bobines embrochables pour les circuits à fréquence $F$.

Le choix du tube d'entrée est primordial dans la recherche de la sensibilité. Cette sensibilité est mise en évidence par le fait que le bruit de la bobine est nettement supérieur au bruit d'entrée du tube (photo 1).

L'amplificateur H.F. a un gain suffisant pour que la détection reste sensiblement linéaire. Son montage est classique avec les précautions habituelles pour éviter la saturation et l'accrochage.

c) Circuit de mise en forme. - Il s'agit de produire des impulsions positives très fines (une microseconde) d'amplitude élevée (quelques centaines de volts) et obtenues aux bornes d'une basse impédance. Nous sommes amenés à adopter le montage ci-dessous ( fig. 4).

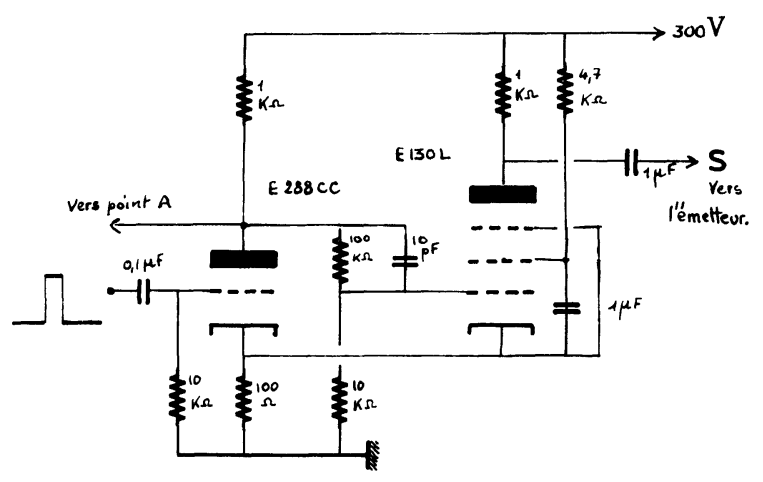

FIG. 4.

Les impulsions, après avoir été mélangées et amplifiées, sont appliquées à l'entrée de ce circuit. On recueille à la sortie des impulsions qui peuvent atteindre $200 \mathrm{~V}$ avec un temps de montée de $100 \mathrm{~ns}$ et une largeur de 300 ns. Cette grande impulsion appliquée à l'écran du tube oscillateur est nécessaire pour produire des impulsions de radiofréquence de quelques centaines de volts. 
d) Dispositif pour supprimer la traînée des impulsions de radiofréquence. - L'un des problèmes le plus général et le plus délicat en échos de spin est l'effet des impulsions de radiofréquence sur le récepteur. Lorsque le temps $T_{2}$ est très court, la décroissance exponentielle de l'impulsion R.F. qui dure un temps supérieur à plusieurs fois la constante de temps $Q / \pi F$ ( $Q$ est le facteur de surtension, $F$ la fréquence d'oscillation) risque de masquer le signal nucléaire. Nous avons réalisé un dispositif très simple et efficace pour supprimer cette traînée ( fig. 5).

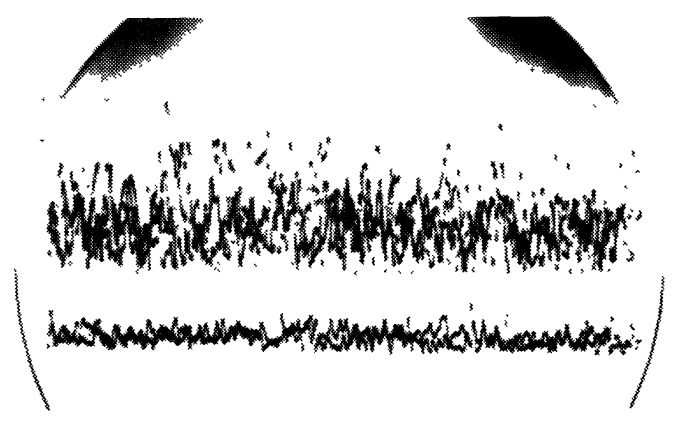

Photographie 1 . - Bruit de la bobine à $45 \mathrm{MHz}$ (en haut) comparé à celui du tube d'entrée (en bas).

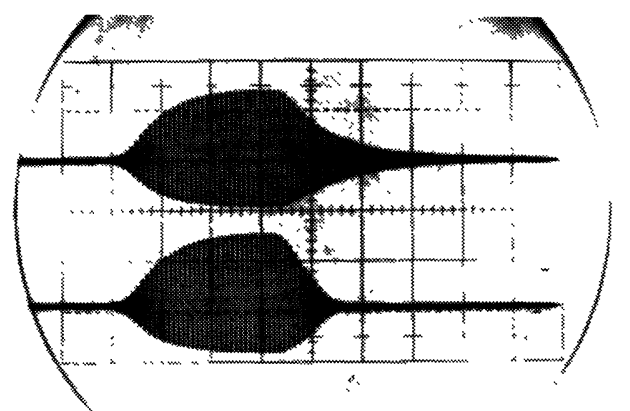

PhOTOGRAPHIE 2. - Impulsions de radiofréquence sans (en haut) et avec (en bas) dispositif pour couper la décroissance exponentielle.

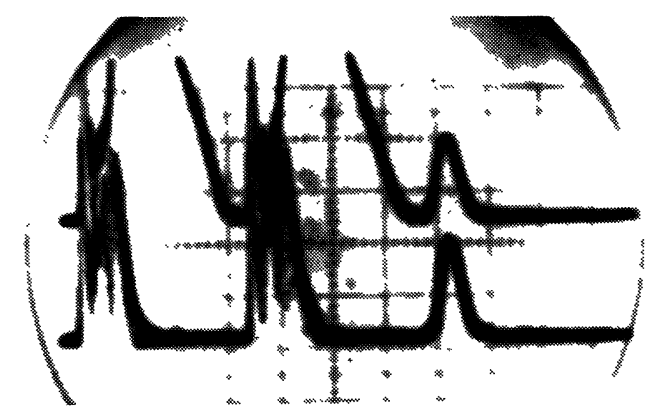

PhóTographie 3. - Écho électronique sans (en haut) et avec (en bas) ce dispositif (1 carreau $=2 \mu \mathrm{s}$ ).

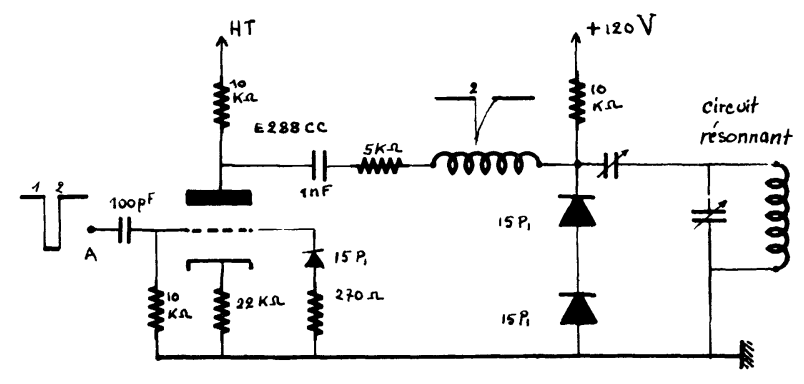

FIG. 5.

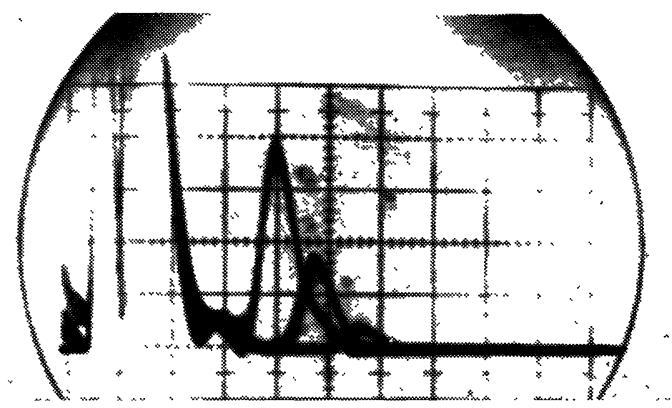

PhotogRaphie 4. - Décroissance de l'écho électronique en fonction de la distance entre les impulsions (1 carreau $=1 \mu \mathrm{s})$.

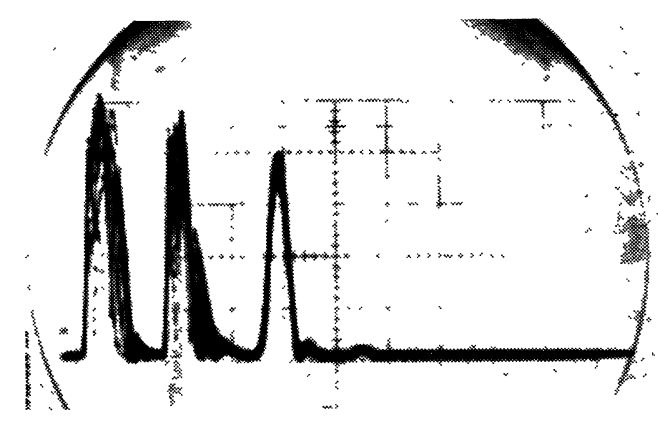

Photographie 5. - Écho électronique secondaire dans le ferrite de nickel (1 carreau $=2 \mu \mathrm{s})$.

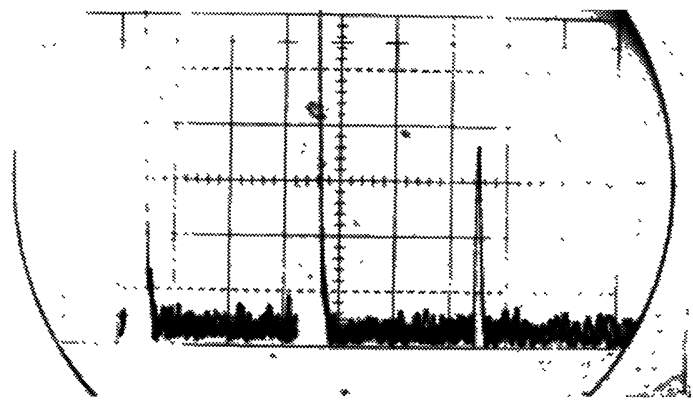

Photographie 6. - Écho nucléaire de ${ }^{57} \mathrm{Fe}$ dans le ferrite de nickel (1 carreau $=10 \mu \mathrm{s})$. 
Le principe est de dériver l'impulsion de manière à obtenir un signal de fin d'impulsion qui permette d'augmenter brusquement l'amortissement du circuit oscillant à cet instant.

Ce dispositif mérite quelques remarques :

1) Le tube $\mathrm{E} 288 \mathrm{CG}$ étant bloqué au repos n'introduit pas de bruit dans le circuit oscillant;

2) La capacité $C_{2}$ doit être réglée soigneusement car elle ajuste l'amortissement du circuit, et on constate que sa valeur optimale est voisine de $20 \mathrm{pF}$ : si elle est trop petite il n'y a pas d'effet, si elle est trop grande les impulsions sont déformées;

3) Le résultat dépend des diodes utilisées. Avec des diodes $15 \mathrm{Pl}$, nous arrivons à rapprocher les impulsions deux fois plus près en présence de l'impulsion négative à l'entrée qu'en son absence;

4) L'impulsion négative est prélevée sur la plaque du tube E 288 GC du circuit de mise en forme;

5) La solution simpliste, souvent utilisée, et qui consiste à diminuer le facteur $Q$ en amortissant le circuit oscillant en régime permanent est catastrophique pour la sensibilité;

6) Ge dispositif n'altère ni la sensibilité, ni le niveau d'oscillation.
Les qualités globales de ce montage ressortent de l'examen de quelques photos d'un écho ayant un temps $T_{2}$ de l'ordre de la microseconde (cet écho observé dans le ferrite de nickel et le grenat d'yttrium entre 30 et $130 \mathrm{MHz}$ est d'origine électronique).

Conclusion. - Nous avons réalisé un appareillage d'écho de spin spécialement conçu pour l'étude de la résonance magnétique nucléaire dans les substances ferro- et ferrimagnétiques, dans la gamme de fréquence 20-150 MHz. Grâce à sa sensibilité et à sa maniabilité, nous avons pu détecter de nouvelles résonances nucléaires dans $\mathrm{CuCr}_{2} \mathrm{O}_{4}$ et $\mathrm{FeCr}_{2} \mathrm{~S}_{4}$ [6]; CrTe et $\mathrm{Cr}_{3} \mathrm{Te}_{4}$ [7]; $\mathrm{CuFe}_{2} \mathrm{O}_{4}, \mathrm{MgFe}_{2} \mathrm{O}_{4}$ [8]; $\mathrm{CoCr}_{2} \mathrm{~S}_{4}$ et $\mathrm{CuCr}_{2} \mathrm{~S}_{4}$ [9]; $\mathrm{Cr}_{2} \mathrm{~S}_{3}$ [10] ainsi que le nickel monodomaine [11]. Ces signaux nucléaires ont des fréquences de résonance situées entre $25 \mathrm{MHz}$ et $110 \mathrm{MHz}$. Soulignons enfin que la quasi-totalité des raies ainsi détectées sont inobservables par la méthode stationnaire.

Remerciements. - Je suis reconnaissant à M. le Professeur P. Grivet qui a bien voulu m'accueillir dans son laboratoire où j'ai pu bénéficier d'excellentes conditions de travail. Il m'est un grand plaisir de remercier MM. J. N. Aubrun et P. Veillet pour leur participation dans la mise au point de cet appareillage.

\section{BIBLIOGRAPHIE}

[1] Hahn (E. L.), Phys. Rev., 1950, 80, 580.

[2] Carr (H. V.) et Purceli, (E. M.), Phys. Rev., 1954, 94, 630.

[3] Poritis (A. M.) et Gossard (A. C.), J. Appl. Phys., Suppl., 1960, 31, 205 S.

[4] Abragam (A.), Les principes du magnétisme nucléaire, p. 37, Institut National des Sciences et Techniques Nucléaires.

[5] KITTeL (C.), Rev. Mod. Phys., 1949, 21, 541.
[6] Le Dang Khor, C. R. Acad. Sc., 1966, 262 B, 1555.

[7] Le Dang Khol et Veirleti (P.), C. R. Acad. Sc., 1967, 264 B, 1154.

[8] Le Dang Khor et Veilitet (P.), C. R. Acad. Sc., 1967, 265 B, 705.

[9] Le Dang KHoI, Solid State Comm., 1968, 6, 203.

[10] Étude en cours.

[11] Aubrun (J. N.) et LE Dang Khoi, C. R. Acad. Sc., 1966, 263 B, 249. 